\title{
Conservative approach for a solitary fibrous tumor of the kidney
}

\author{
Alberto Martini ${ }^{1}$, Eugenio Dattolo ${ }^{1}$, Maria Rosaria Raspollini ${ }^{2}$, Donata Villari ${ }^{1}$, Giulio Nicita ${ }^{1}$, Maria Cristina Paoletti ${ }^{1}$ \\ ${ }^{1}$ Department of Urology, University of Florence, Careggi Hospital, Florence - Italy \\ ${ }^{2}$ Histopathology and Molecular Diagnostics, University of Florence, Careggi Hospital, Florence - Italy
}

\begin{abstract}
Introduction: Solitary fibrous tumor (SFT) of the kidney represents a rare neoplasm, and its malignant potential seems to be scarce. Classically, a radical nephrectomy is regarded as the treatment of choice for this condition.

Case report: We present the case of a 37-year-old male patient who underwent left robotic clampless partial nephrectomy of an SFT of the kidney. Our patient has been followed-up carefully, and at 15-month examination, he is free of recurrence. This is the second case of an SFT managed through a conservative approach.

Conclusions: In our opinion, according to tumor's dimensions, partial nephrectomy may be considered for treating small SFTs.
\end{abstract}

Keywords: Conservative treatment, Oncological outcome, Robotic partial nephrectomy, Solitary fibrous tumor

\section{Introduction}

Solitary fibrous tumors (SFTs) are unusual spindle cell neoplasms that typically occur in the pleura but have been described in extrapleural sites (1). Urogenital localization is rare; the first case of SFT of the kidney was described in 1996 (2). The diagnosis of SFTs relies on pathological examination (3). They can present either malignant or benign characteristics at pathological examination. Despite these features, the malignant potential of SFTs seems to be scarce with evidence of only four cases of metastasis in literature (4-7). In one case only, out of more than 60 reported, the tumor manifested extracapsular extension toward renal parenchyma (7).

Classically, a radical nephrectomy is regarded as the treatment of choice for treating this condition; there is evidence of a conservative approach in one case only, in which a solitary kidney patient underwent partial nephrectomy along with autotransplantation (8). The aim of this paper is to report our approach for treating a renal SFT. To the best of our knowledge, this is the second case of an SFT managed through a conservative approach.

Accepted: August 29, 2016

Published online: September 27, 2016

\section{Corresponding author:}

Alberto Martini, MD

Clinica Urologica II, Azienda Ospedaliera Careggi

Università di Firenze, Viale San Luca

50134 Firenze, Italy

a.martini.md@gmail.com

\section{Case description}

A 37-year-old male patient underwent left laparoscopic robotic-assisted partial nephrectomy of an SFT of the kidney. The tumor was incidentally found at computed tomography (CT) scan. This showed a well-circumscribed $2 \mathrm{~cm}$ mass with almost no enhancement change during arterial and venous phase, Fig. $1 A$ and $B$, respectively.

According to the mass characteristics (almost no enhancement change due to high collagen content, completely exophytic growth, and well-circumscribed lesion), the radiologist had hypothesized an SFT. Given tumor's location and dimensions, a biopsy has been ruled out. The patient was scheduled for a robotic partial nephrectomy. As the tumor manifested with exophytic growth, a clampless approach has been adopted.

A three-arm transperitoneal approach, using $30^{\circ}$ lens, was chosen.

After general endotracheal anesthesia was administered, the patient was placed in full flank position. The first Trocar $(12 \mathrm{~mm})$ was positioned, according to the Hasson technique, $2 \mathrm{~cm}$ above the umbilicus on the pararectal line. The remaining two $(8 \mathrm{~mm})$ trocars were placed superiorly and inferiorly, respectively, to the camera trocar under direct vision. One surgeon through a $12 \mathrm{~mm}$ port, placed along the pararectal line, assisted the console surgeon laparoscopically. The da Vinci Si robot was docked perpendicularly to the patient.

The plan between the tumor and the renal parenchyma was bluntly and sharply developed using Maryland bipolar forceps and monopolar curved scissors. Careful attention was paid in handling the dissection in order to avoid any accidental damage to the renal parenchyma. As the tumor margins 

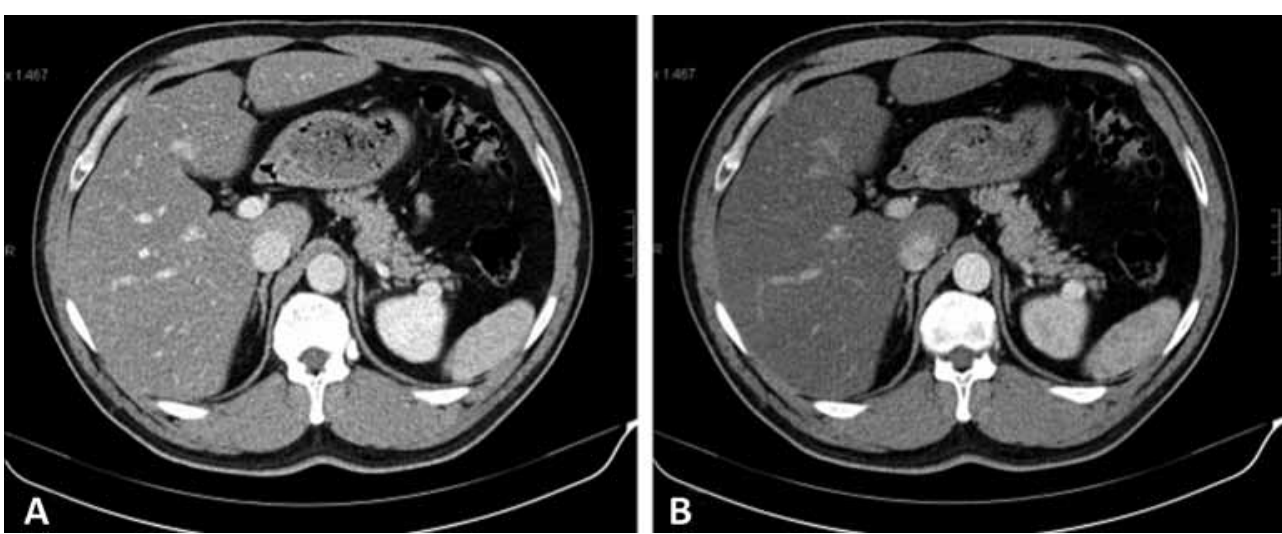

Fig. 1 - CT scan of the abdomen. (A) Arterial phase. (B) Venous phase.

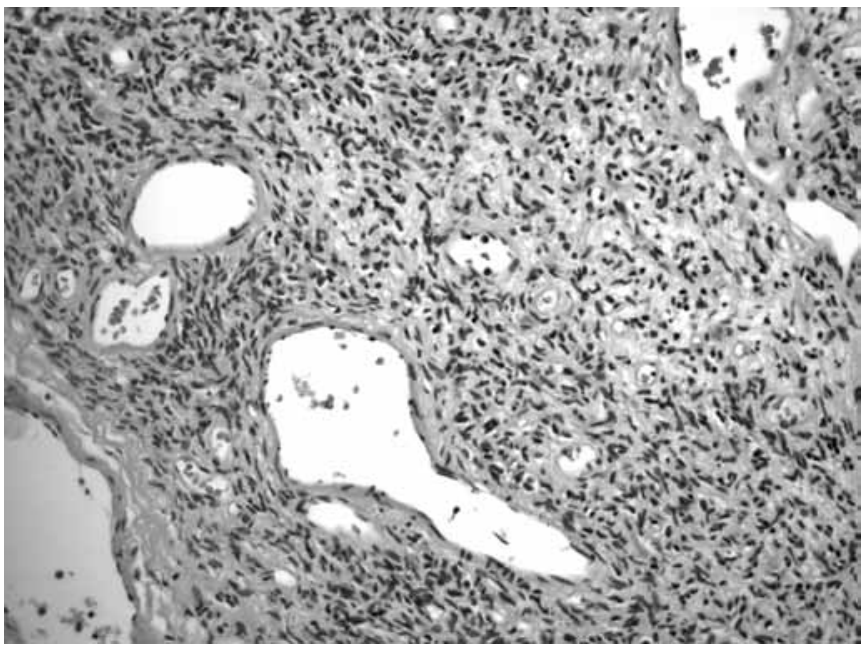

Fig. 2 - Spindle cells with collagenous bands and vessels. Hematoxylin-eosin staining.

were well demarcated, there was no need for renorraphy. Floseal ${ }^{\circledR}$ application, at the resection bed completed the operation. After dissection, the specimen was removed intact through the camera port using a retrieval bag.

Total operative time was 120 minutes, while console time was 90 minutes. Estimated blood loss was $50 \mathrm{ml}$. The postoperative course was uneventful and the patient was discharged on the fifth postoperative day. The patient has been followedup carefully with ultrasound and CT scan; at 15-month followup examination, he is free of recurrence.

\section{Histopathologic analysis}

The macroscopic evaluation showed the presence of a circumscribed, solid, white-grey mass of $2.5 \times 2.5 \mathrm{~cm}$. The specimen was fixed in $10 \%$ neutral buffered formalin, and representative sections of the renal mass were embedded in paraffin, sectioned in 3- to 4-micron thickness, and stained with hematoxylin-eosin. Other histological sections were performed for immunohistochemical analysis.

Microscopic evaluation showed the presence of an encapsulated tumor constituted by spindle cells with storiform or fascicular arrangements (Figs. 2, 3).

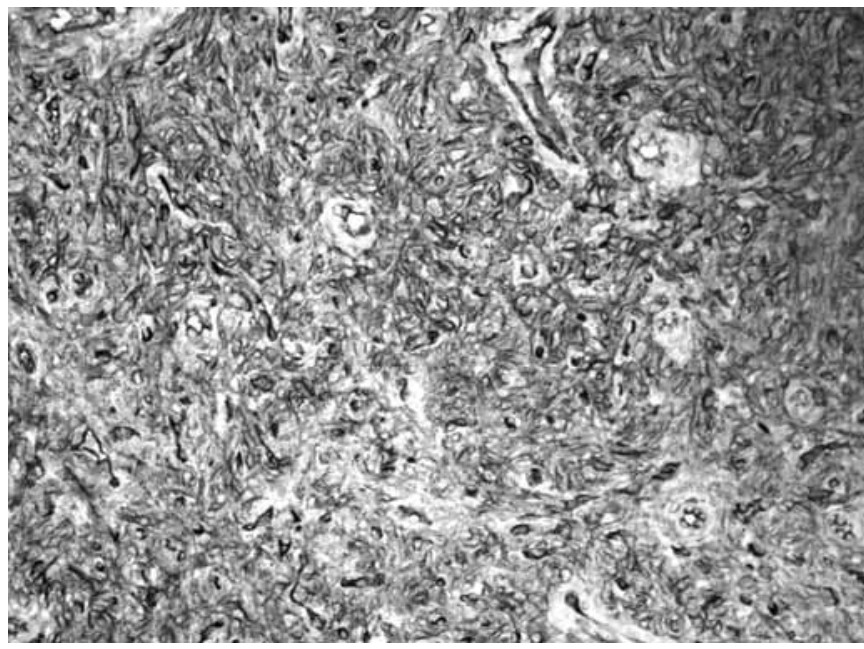

Fig. 3 - Diffuse and strong cytoplasmatic CD34 staining in the tumor cells.

The spindle cell showed a plump and bland nucleous and a pale cytoplasm. Mitotic figures were uncommon. Areas with vessels were present. No necrosis was observed. The tumor was linked to the renal capsule. Immunohistochemical evaluation showed CD34 positivity (Fig. 3), while CD31, Actin 1A4, CD117, S100, MART1, CD10, CAM5.2, and CK7 were negative. Ki 67 was positive in $5 \%$ of the tumor cells. The microscopic features and the positive immunohistochemical staining for CD34 led to the diagnosis of an SFT, confirming radiologist's initial suspect.

\section{Conclusion}

SFT is a rare neoplasm. Its diagnosis relies on histopathological examination. High positivity for CD34 is useful for differentiating SFTs from other spindle cells tumors. In fact, CD34, which is a stem-cell adhesion marker, is considered a specific immune-peroxidase marker for SFTs (1). Pathological criteria for malignancy include increased cellularity, pleomorphism, increased mitotic activity (more than four mitoses on 10 high power fields), necrosis, and hemorrhage and a $20 \% \mathrm{Ki}-67$ proliferative index (3). Despite histopathological characteristics, metastatic behavior, among malignant tumors, has been reported in four cases only (4-7), while local invasion in one (7). 
Metastasis have been described occurring to pancreas (4), lungs $(5,6)$, and vertebrae. (7) Regarding invasive behavior, in one case, the tumor manifested local invasion toward the renal capsule and hilum (7). Although the clinical course of SFTs can be unpredictable, the prognosis is generally favorable (1).

Concerning clinical diagnosis, similarly to renal cell carcinoma, SFTs of the kidney may manifest with the triad of flank pain, a palpable abdominal mass, and gross hematuria (1). Paraneoplastic syndromes from SFTs are rare; nevertheless, hypoglycemia has been described in two cases $(9,10)$ due to insulin-like growth factor-2 (IGF-2) overproduction; histological malignancy was reported in both cases $(9,10)$.

Kather et al, in their review, reported that there is no evidence whether a conservative approach, through partial nephrectomy, has the same oncological outcome as radical nephrectomy for treating SFTs (1). In one previous case only, a partial nephrectomy along with autotransplantation in a solitary kidney patient has been performed (8). The patient is reported to be free of disease at 1-year follow-up (8).

Our patient's tumor resulted without clear malignant histopathological features. The patient is free of disease at 15-month follow-up examination.

In literature, it is reported that there is no evidence whether a conservative approach for treating SFTs has the same oncological outcome as radical nephrectomy. Given the rarity of this condition, it is not easy to demonstrate what is the best approach for SFTs treatment.

As partial nephrectomy has become routine for treating small renal mass in case of renal cell carcinoma, tumor that has a more malignant potential than SFTs, a conservative approach may be attempted in case of small SFTs, especially when such diagnosis is preoperatively suspected and/or biopsy confirmed.

\section{Disclosures}

Financial support: The authors declared that this study has received no financial support.
Conflict of interest: No conflict of interest was declared by the authors.

\section{References}

1. Khater N, Khauli R, Shahait M, Degheili J, Khalifeh I, Aoun J. Solitary fibrous tumors of the kidneys: presentation, evaluation, and treatment. Urol Int. 2013;91(4):373-383.

2. Gelb $A B$, Simmons $M L$, Weidner N. Solitary fibrous tumor involving the renal capsule. Am J Surg Pathol. 1996;20(10): 1288-1295.

3. Guillou LFJ, Fletcher CDM, Mandahi N. (2001) Extrapleural solitary fibrous tumour and hemangiopericytoma; in Fletcher CDM, Unni KK, Mertens F (ed): World Health Organization Classification of Tumours: Pathology and Genetics of Tumours of Soft Tissue and Bone. Lyon, IARC Press pp 86-90.

4. Patel YA, Dhalla S, Olson MT, Lennon AM, Khashab MA, Singh VK. Pancreatic metastasis from a solitary fibrous tumor of the kidney: a rare cause of acute recurrent pancreatitis. Pancreatology. 2013;13(6):631-633.

5. Fine SW, McCarthy DM, Chan TY, Epstein JI, Argani P. Malignant solitary fibrous tumor of the kidney: report of a case and comprehensive review of the literature. Arch Pathol Lab Med. 2006;130(6):857-861.

6. Cuello J, Brugés R. Malignant solitary fibrous tumor of the kidney: report of the first case managed with interferon. Case Rep Oncol Med. 2013;2013:564980.

7. Guo G, Zhang X, Zhou ZH. Clinical characteristics of malignant solitary fibrous tumors of the kidney with thoracic vertebral metastasis. Int J Urol. 2012;19(2):177-178.

8. Makris A, Tabaza R, Brehmer B, Lindemann-Docter K, Wildberger J, Jakse G. Solitary fibrous tumor of the kidney: a case report. Can J Urol. 2009;16(5):4854-4856.

9. Zhao G, Li G, Han R. Two malignant solitary fibrous tumors in one kidney: Case report and review of the literature. Oncol Lett. 2012;4(5):993-995.

10. Khowaja A, Johnson-Rabbett B, Bantle J, Moheet A. Hypoglycemia mediated by paraneoplastic production of Insulin like growth factor-2 from a malignant renal solitary fibrous tumor - clinical case and literature review. BMC Endocr Disord. 2014;14(1):49. 